\title{
CONSORT 2010 Statement: updated guidelines for reporting parallel group randomized trials
}

\author{
Kenneth F Schulz, Douglas G Altman, David Moher; for the CONSORT Group
}

Kenneth F Schulz is distinguished scientist and vice president, Family Health International, Research Triangle Park, North Carolina, U.S. Douglas G Altman is professor, Centre for Statistics in Medicine, University of Oxford, Wolfson College, Oxford, England. David Moher is senior scientist, Ottawa Methods Centre, Clinical Epidemiology Program, Ottawa Hospital Research Institute, Department of Epidemiology and Community Medicine, University of Ottawa, Ottawa, Ontario, Canada.

Funding: We received financial support from United Kingdom National Institute for Health Research and the Medical Research Council; Canadian Institutes of Health Research; Presidents Fund, Canadian Institutes of Health Research; Johnson \& Johnson; BMJ; and the American Society for Clinical Oncology. Douglas Altman is supported by Cancer Research UK, David Moher by a University of Ottawa Research Chair and Kenneth Schulz by Family Health International. None of the sponsors had any involvement in the planning, execution or writing of the CONSORT documents. Additionally, no funder played a role in drafting the manuscript.

Competing interests: Uniform disclosure of potential conflicts of interest: all authors have completed the ICMJE unified competing interest form at www.icmje.org/coi_disclosure.pdf (available from the corresponding author) and declare (1) David Moher received grants for this work from Johnson \& Johnson, BMJ, and American Society for Clinical Oncology; Kenneth Schulz and Douglas Altman received support for travel to meetings for this work from Johnson \& Johnson, BMJ, and American Society for Clinical Oncology; (2) Kenneth Schulz and Douglas Altman had travel expenses reimbursed by the EQUATOR Network; Kenneth Schulz has received honoraria for delivering educational presentations for the American Board of Obstetrics and Gynecology Foundation for Excellence in Women's Health Care, Ortho-McNeil Janssen Scientific Affairs, and the American College of Obstetrics and Gynecology; and has done consultancy for Wyeth. All authors also declare (3) no spouses, partners, or children with relationships with commercial entities that might have an interest in the submitted work; (4) no non-financial interests that may be relevant to the submitted work.

Correspondence: K F Schulz, kschulz@fhi.org

$\mathrm{R}$ ANDOMIZED CONTROLLED TRIALS, WHEN THEY are appropriately designed, conducted and ating health care interventions. However, randomized trials can yield biased results if they lack methodological rigour. ${ }^{1}$ To assess a trial accurately, readers of a published report need complete, clear and transparent information on its methodology and findings. Unfortunately, attempted assessments frequently fail because authors of many trial reports neglect to provide lucid and complete descriptions of that critical information. ${ }^{2-4}$

That lack of adequate reporting fuelled the development of the original CONSORT (Consolidated Standards of Reporting Trials) statement in $1996^{5}$ and its revision 5 years later. ${ }^{6-8}$ While those statements improved the reporting quality for some randomized controlled trials, 9,10 many trial reports still remain inadequate. ${ }^{2}$ Furthermore, new methodological evidence and additional experience has accumulated since the last revision in 2001. Consequently, we organized a CONSORT Group meeting to update the 2001 statement. ${ }^{6-8}$ We introduce here the result of that process, CONSORT 2010.

\section{Intent of CONSORT 2010}

The CONSORT 2010 Statement is this paper including the 25-item checklist in the table (see Table 1) and the flow diagram (see Fig. 1). It provides guidance for reporting all randomized controlled trials, but focuses on the most common design type-individually randomized, 2-group parallel trials. Other trial designs, such as cluster randomized trials and non-inferiority trials, require varying amounts of additional information. CONSORT extensions for these designs, ${ }^{11,12}$ and other CONSORT products, can be found through the CONSORT website (www.consort-statement.org). Along with the CONSORT statement, we have updated the explanation and elaboration article, ${ }^{13}$ which explains the inclusion of each checklist item, provides methodological background and gives published examples of transparent reporting.

Diligent adherence by authors to the checklist items facilitates clarity, completeness and transparency of reporting. Explicit descriptions, not ambiguity or omission, best serve the interests of all readers. Note that the 


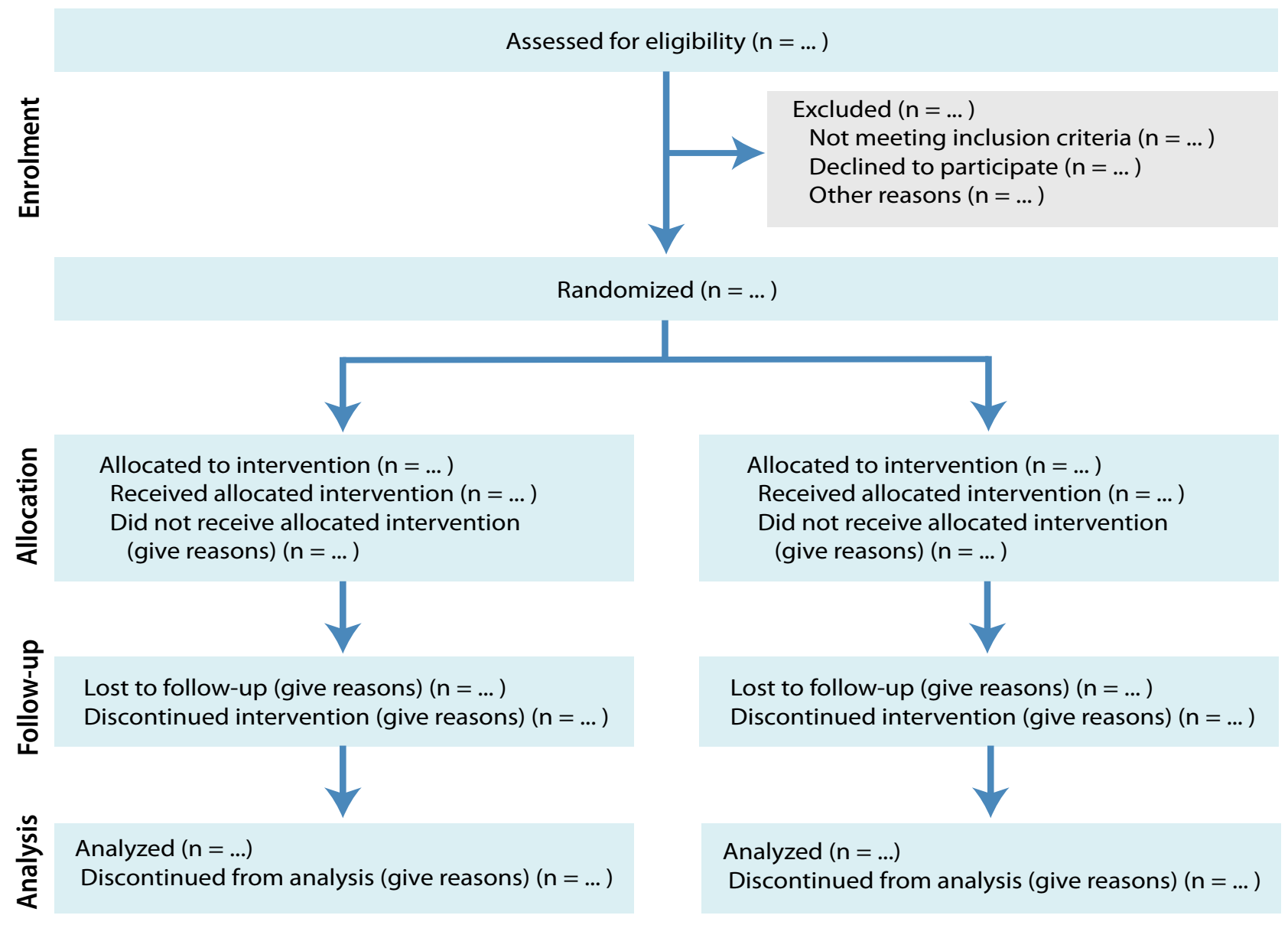

Figure 1: Flow diagram of the progress through the phases of a parallel randomized trial of 2 groups (that is, enrolment, intervention allocation, follow-up, and data analysis).

CONSORT 2010 Statement does not include recommendations for designing, conducting and analyzing trials. It solely addresses the reporting of what was done and what was found.

Nevertheless, CONSORT does indirectly affect design and conduct. Transparent reporting reveals deficiencies in research if they exist. Thus, investigators who conduct inadequate trials, but who must transparently report, should not be able to pass through the publication process without revelation of their trial's inadequacies. That emerging reality should provide impetus to improved trial design and conduct in the future, a secondary indirect goal of our work. Moreover, CONSORT can help researchers in designing their trial.

\section{Background to CONSORT}

Efforts to improve the reporting of randomized controlled trials accelerated in the mid-1990s, spurred partly by methodological research. Researchers had shown for many years that authors reported such trials poorly, and empirical evidence began to accumulate that some poorly conducted or poorly reported aspects of trials were associated with bias. ${ }^{14}$ Two initiatives aimed at developing reporting guidelines culminated in one of us (DM) and Drummond Rennie organizing the first CONSORT statement in 1996. ${ }^{5}$ Further methodological research on similar topics reinforced earlier findings $\mathrm{s}^{15}$ and fed into the revision of 2001. ${ }^{6-8}$ Subsequently, the expanding body of methodological research informed the refinement of CONSORT 2010. More than 700 studies comprise the CONSORT database (located on the CONSORT website), which provides the empirical evidence to underpin the CONSORT initiative.

Indeed, CONSORT Group members continually monitor the literature. Information gleaned from these efforts provides an evidence base on which to update the CONSORT statement. We add, drop or modify items based on that evidence and the recommendations of the CONSORT Group, an international and eclectic group of clinical trialists, statisticians, epidemiologists and biomedical editors. The CONSORT Executive (KFS, DGA, DM) strives for a balance of established and emerging 
researchers. The membership of the group is dynamic. As our work expands in response to emerging projects and needed expertise, we invite new members to contribute. As such, CONSORT continually assimilates new ideas and perspectives. That process informs the continually evolving CONSORT statement.

Over time, CONSORT has garnered much support. More than 400 journals, published around the world and in many languages, have explicitly supported the CONSORT statement. Many other health care journals support it without our knowledge. Moreover, thousands more have implicitly supported it with the endorsement of the CONSORT statement by the International Committee of Medical Journal Editors (www.icmje.org). Other prominent editorial groups, the Council of Science Editors and the World Association of Medical Editors, officially support CONSORT. That support seems warranted: when used by authors and journals, CONSORT seems to improve reporting. ${ }^{9}$

\section{Development of CONSORT 2010}

Thirty-one members of the CONSORT 2010 Group met in Montebello, Canada, in January 2007 to update the 2001 CONSORT statement. In addition to the accumulating evidence relating to existing checklist items, several new issues had come to prominence since 2001. Some participants were given primary responsibility for aggregating and synthesizing the relevant evidence on a particular checklist item of interest. Based on that evidence, the group deliberated the value of each item. As in prior CONSORT versions, we kept only those items deemed absolutely fundamental to reporting a randomized controlled trial. Moreover, an item may be fundamental to a trial but not included, such as approval by an institutional and meetings to revise the checklist. After 7 major iterations, a revised checklist was distributed to the larger group for feedback. With that feedback, the executive met twice in person to consider all the comments and to produce a penultimate version. That served as the basis for writing the first draft of this paper, which was then distributed to the group for feedback. After consideration of their comments, the executive finalized the statement.

The CONSORT Executive then drafted an updated explanation and elaboration manuscript, with assistance from other members of the larger group. The substance of the 2007 CONSORT meeting provided the material for the update. The updated explanation and elaboration manuscript was distributed to the entire group for additions, deletions and changes. That final iterative process converged to the CONSORT 2010 Explanation and Elaboration. ${ }^{13}$

\section{Changes in CONSORT 2010}

The revision process resulted in evolutionary, not revolutionary, changes to the checklist (Table 1), and the flow diagram was not modified except for 1 word (Fig. 1). Moreover, because other reporting guidelines augmenting the checklist refer to item numbers, we kept the existing items under their previous item numbers except for some renumbering of items 2 to 5 . We added additional items either as a sub-item under an existing item, an entirely new item number at the end of the checklist, or (with item 3) an interjected item into a renumbered segment. We have summarized the noteworthy general changes in Box 1 and specific changes in Box 2. The CONSORT website contains a side-by-side comparison of the 2001 and 2010 versions. ethical review board, because funding bodies strictly enforce ethical review and medical journals usually address reporting ethical review in their instructions for authors. Other items may seem desirable, such as reporting on whether on-site monitoring was done, but a lack of empirical evidence or any consensus on their value cautions against inclusion at this point. The CONSORT 2010 Statement thus addresses the minimum criteria, although that should not deter authors from including other information if they consider it important.

After the meeting, the CONSORT Executive convened teleconferences

\section{Box 1: Noteworthy general changes in CONSORT 2010 Statement}

- We simplified and clarified the wording, such as in items $1,8,10,13,15,16,18$, 19 and 21.

- We improved consistency of style across the items by removing the imperative verbs that were in the 2001 version.

- We enhanced specificity of appraisal by breaking some items into sub-items. Many journals expect authors to complete a CONSORT checklist indicating where in the manuscript the items have been addressed. Experience with the checklist noted pragmatic difficulties when an item comprised multiple elements. For example, item 4 addresses eligibility of participants and the settings and locations of data collection. With the 2001 version, an author could provide a page number for that item on the checklist, but might have reported only eligibility in the paper, for example, and not reported the settings and locations. CONSORT 2010 relieves obfuscations and forces authors to provide page numbers in the checklist for both eligibility and settings. 


\section{Implications and limitations}

We developed CONSORT 2010 to assist authors in writing reports of randomized controlled trials, editors and peer reviewers in reviewing manuscripts for publication, and readers in critically appraising published articles. The CONSORT 2010 Explanation and Elaboration provides elucidation and context to the checklist items. We strongly recommend using the explanation and elaboration in conjunction with the checklist to foster complete, clear and transparent reporting and aid appraisal of published trial reports.

CONSORT 2010 focuses predominantly on the 2-group, parallel randomized controlled trial, which accounts for over half of trials in the literature. ${ }^{2}$ Most of the items from the CONSORT 2010 Statement, however, pertain to all types of randomized trials. Nevertheless, some types of trials or trial situations dictate the need for additional information in the trial report. When in doubt, authors, editors and readers should consult the CONSORT website for any CONSORT extensions, expansions (amplifications), implementations or other guidance that may be relevant.

The evidence-based approach we have used for CONSORT also served as a model for development of other reporting guidelines, such as for reporting systematic reviews and meta-analyses of studies evaluating interventions, ${ }^{16}$ diagnostic studies, ${ }^{17}$ and observational studies. ${ }^{18}$ The explicit goal of all these initiatives is to improve reporting. The Enhancing the Quality and Transparency of Health Research (EQUATOR) Network will facilitate development of reporting guidelines and help disseminate the guidelines: www.equator-network.org provides information on all reporting guidelines in health research.

With CONSORT 2010, we again intentionally declined to produce a rigid structure for the reporting of randomized trials. Indeed, SORT ${ }^{19}$ tried a rigid format, and it failed in a pilot run with an editor and authors. ${ }^{20}$ Consequently, the format of articles should abide by journal style, editorial directions, the traditions of the research field addressed, and, where possible, author preferences. We do not wish to standardize the structure of reporting. Authors should simply address checklist items somewhere in the article, with ample detail and lucidity. That stated, we think that manuscripts benefit from frequent subheadings within the major sections, especially the methods and results sections.

CONSORT urges completeness, clarity and transparency of reporting, which simply reflects the actual trial design and conduct. However, as a potential drawback, a reporting guideline might encourage some authors to report fictitiously the information suggested by the guidance rather than what was actually done. Authors, peer reviewers and editors should vigilantly guard against that potential drawback and refer, for example, to trial protocols, to information on trial registers and to regulatory agency websites. Moreover, the CONSORT 2010 Statement does not include recommendations for designing and conducting randomized trials. The items should elicit clear pronouncements of how and what the authors did, but do not contain any judgments on how and what the authors should have done. Thus, CONSORT 2010 is not intended as an instrument to evaluate the quality of a trial. Nor is it appropriate to use the checklist to construct a "quality score."

Nevertheless, we suggest that researchers begin trials with their end publication in mind. Poor reporting allows authors, intentionally or inadvertently, to escape scrutiny of any weak aspects of their trials. However, with wide adoption of CONSORT by journals and editorial groups, most authors should have to report transparently all important aspects of their trial. The ensuing scrutiny rewards well-conducted trials and penalizes poorly conducted trials. Thus, investigators should understand the CONSORT 2010 reporting guidelines before starting a trial as a further incentive to design and conduct their trials according to rigorous standards.

CONSORT 2010 supplants the prior version published in 2001. Any support for the earlier version accumulated from journals or editorial groups will automatically extend to this newer version, unless specifically requested otherwise. Journals that do not currently support CONSORT may do so by registering on the CONSORT website. If a journal supports or endorses CONSORT 2010, it should cite one of the original versions of CONSORT 2010, the CONSORT 2010 Explanation and Elaboration, and the CONSORT website in their "Instructions to authors." We suggest that authors who wish to cite CONSORT should cite this or another of the original journal versions of CONSORT 2010 Statement, and, if appropriate, the CONSORT 2010 Explanation and Elaboration. ${ }^{13}$ All CONSORT material can be accessed through the original publishing journals or the CONSORT website. Groups or individuals who desire to translate the CONSORT 2010 Statement into other languages should first consult the CONSORT policy statement on the website.

We emphasize that CONSORT 2010 represents an evolving guideline. It requires perpetual reappraisal and, if necessary, modifications. In the future we will further revise the CONSORT material considering comments, criticisms, experiences and accumulating new evidence. We invite readers to submit recommendations via the CONSORT website. 


\section{Box 2: Noteworthy specific changes in CONSORT 2010 Statement}

\begin{tabular}{|ll} 
Item $1 \mathrm{~b}$ (title and abstract) & $\begin{array}{l}\text { We added a sub-item on providing a structured summary of trial design, methods, results, and } \\
\text { conclusions and referenced the CONSORT for abstracts article }{ }^{21}\end{array}$ \\
\hline Item $2 \mathrm{~b}$ (introduction) & We added a new sub-item (formerly item 5 in CONSORT 2001) on "Specific objectives or hypotheses" \\
\hline Item $3 \mathrm{a}$ (trial design) & $\begin{array}{l}\text { We added a new item including this sub-item to clarify the basic trial design (such as parallel group, } \\
\text { crossover, cluster) and the allocation ratio }\end{array}$
\end{tabular}

Item 3b (trial design)

We added a new sub-item that addresses any important changes to methods after trial commencement, with a discussion of reasons

Item 4 (participants

Formerly item 3 in CONSORT 2001

Item 5 (interventions)

Formerly item 4 in CONSORT 2001. We encouraged greater specificity by stating that descriptions of interventions should include "sufficient details to allow replication"3

Item 6 (outcomes)

We added a sub-item on identifying any changes to the primary and secondary outcome (endpoint) measures after the trial started. This followed from empirical evidence that authors frequently provide analyses of outcomes in their published papers that were not the pre-specified primary and secondary outcomes in their protocols, while ignoring their pre-specified outcomes (that is, selective outcome reporting). ${ }^{4,22}$ We eliminated text on any methods used to enhance the quality of measurements

Item 9 (allocation concealment

We reworded this to include mechanism in both the report topic and the descriptor to reinforce mechanism) that authors should report the actual steps taken to ensure allocation concealment rather than simply report imprecise, perhaps banal, assurances of concealment

Item 11 (blinding)

We added the specification of how blinding was done and, if relevant, a description of the similarity of interventions and procedures. We also eliminated text on "how the success of blinding (masking) was assessed" because of a lack of empirical evidence supporting the practice as well as theoretical concerns about the validity of any such assessment ${ }^{23,24}$

Item 12a (statistical methods) We added that statistical methods should also be provided for analysis of secondary outcomes Sub-item 14b (recruitment) Based on empirical research, we added a sub-item on "Why the trial ended or was stopped"25

Item 15 (baseline data)

We specified "A table" to clarify that baseline and clinical characteristics of each group are most clearly expressed in a table

Item 16 (numbers analyzed)

We replaced mention of "intention to treat" analysis, a widely misused term, by a more explicit request for information about retaining participants in their original assigned groups ${ }^{26}$

Sub-item 17b (outcomes and For appropriate clinical interpretability, prevailing experience suggested the addition of "For binary estimation) outcomes, presentation of both relative and absolute effect sizes is recommended"27

Item 19 (harms)

We included a reference to the CONSORT paper on harms ${ }^{28}$

Item 20 (limitations)

We changed the topic from "Interpretation" and supplanted the prior text with a sentence focusing on the reporting of sources of potential bias and imprecision

Item 22 (interpretation)

We changed the topic from "Overall evidence." Indeed, we understand that authors should be allowed leeway for interpretation under this nebulous heading. However, the CONSORT Group expressed concerns that conclusions in papers frequently misrepresented the actual analytical results and that harms were ignored or marginalized. Therefore, we changed the checklist item to include the concepts of results matching interpretations and of benefits being balanced with harms

Item 23 (registration)

We added a new item on trial registration. Empirical evidence supports the need for trial registration, and recent requirements by journal editors have fostered compliance ${ }^{29}$

Item 24 (protocol)

We added a new item on availability of the trial protocol. Empirical evidence suggests that authors often ignore, in the conduct and reporting of their trial, what they stated in the protocol..$^{4,22}$ Hence, availability of the protocol can instigate adherence to the protocol before publication and facilitate assessment of adherence after publication

Item 25 (funding)

We added a new item on funding. Empirical evidence points toward funding source sometimes being associated with estimated treatment effects ${ }^{30}$ 


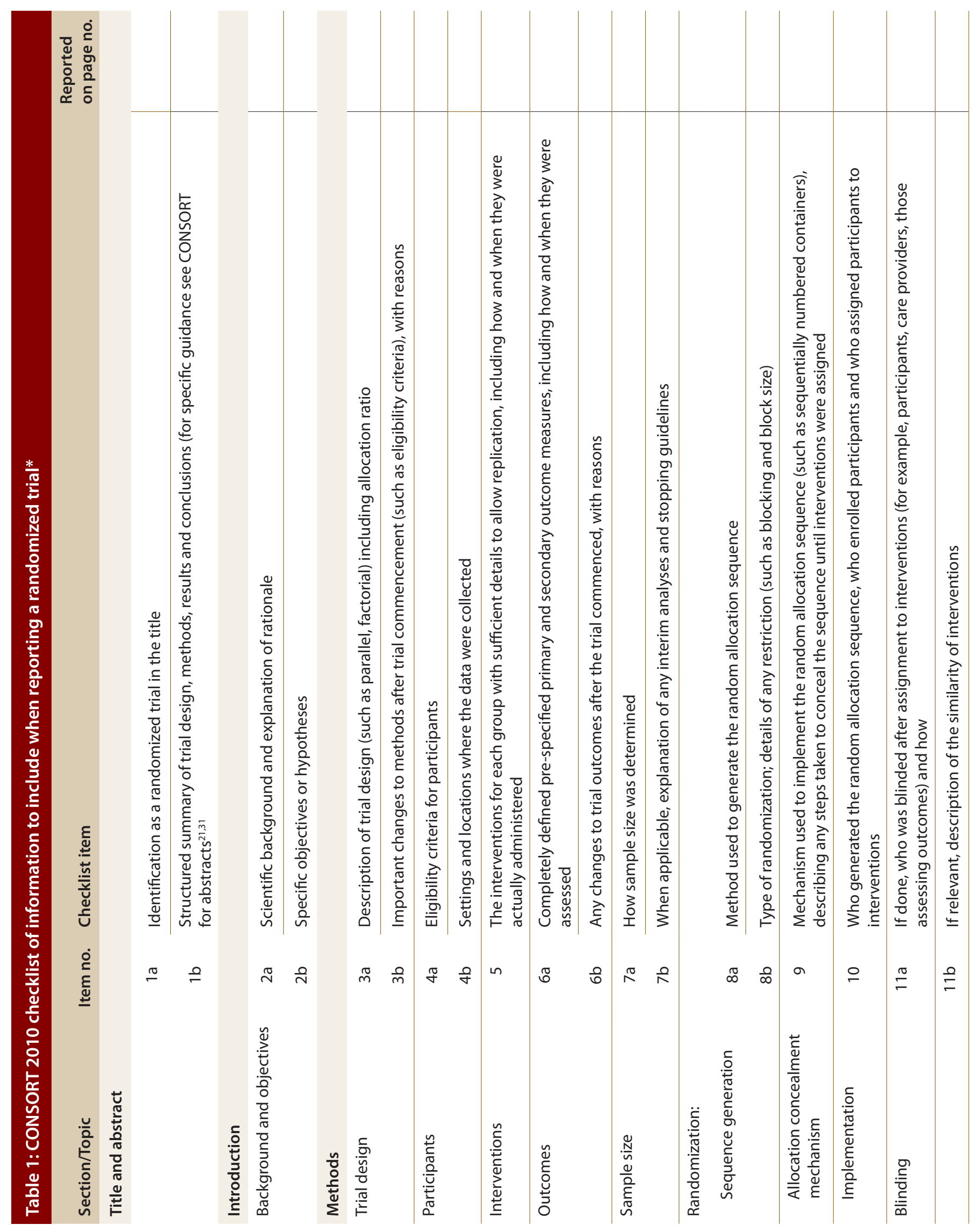




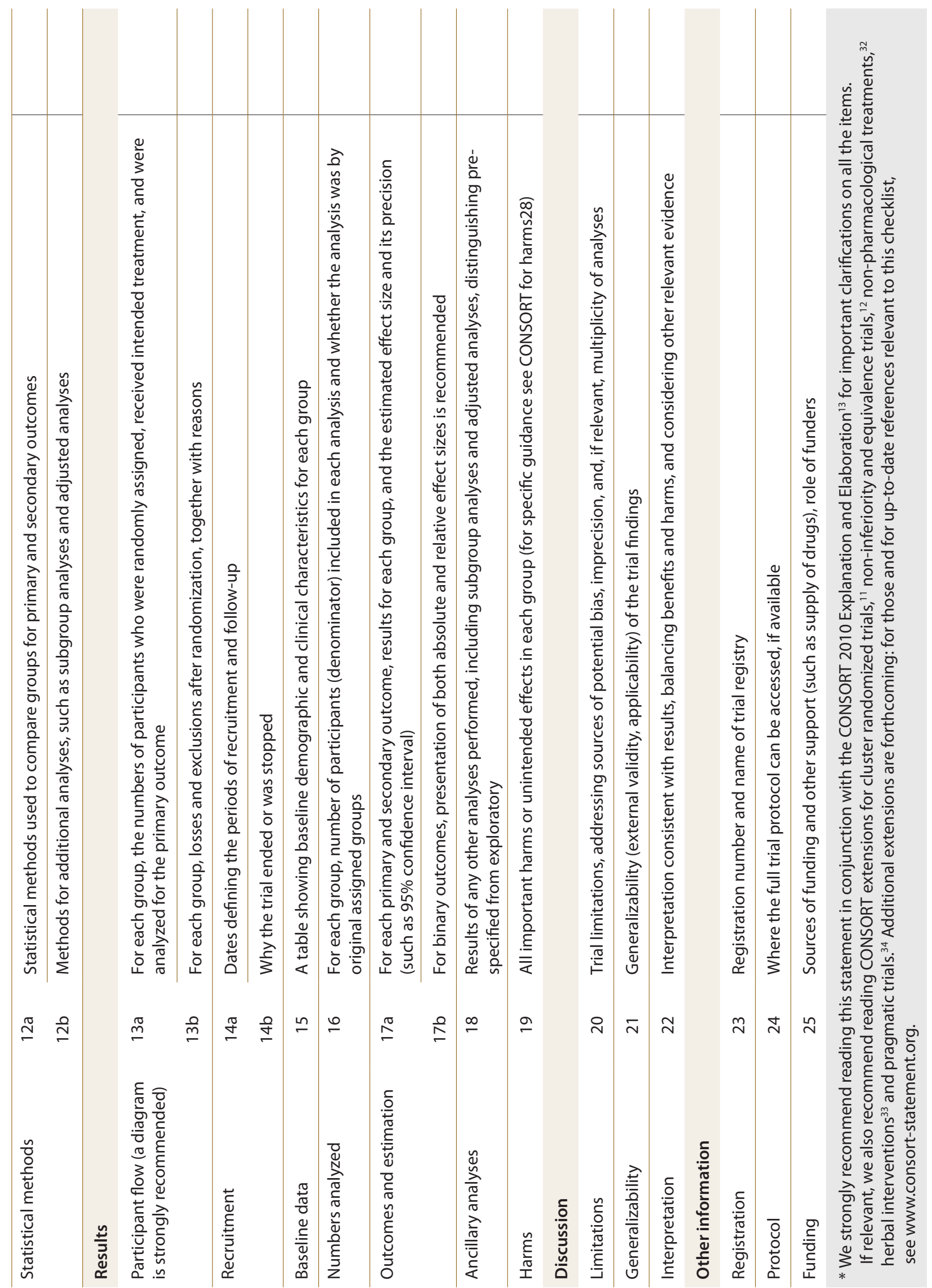


Contributors: Kenneth Schulz, David Moher and Douglas Altman participated in meetings and regular conference calls, planned the' CONSORT 2007 meeting at Montebello, developed the agenda, prepared background research, identified and invited participants, contributed to the CONSORT meeting, drafted the manuscript, and, after critical review by the CONSORT Group, finalized the text of the manuscript. Members of the CONSORT Group attended the meeting, except for those noted below, and provided input on and review of the revised checklist and text of this article. Some members also prepared background material.

The CONSORT Group contributors to CONSORT 2010: DG Altman, Centre for Statistics in Medicine, University of Oxford; Virginia Barbour, PLoS Medicine; Jesse A Berlin, Johnson \& Johnson Pharmaceutical Research and Development, USA; Isabelle Boutron, University Paris 7 Denis Diderot, Assistance Publique des Hôpitaux de Paris, INSERM, France; PJ Devereaux, MCMaster University, Canada; Kay Dickersin, Johns Hopkins Bloomberg School of Public Health, US; Diana Elbourne, London School of Hygiene \& Tropical Medicine; Susan Ellenberg, University of Pennsylvania School of Medicine, US; Val Gebski, University of Sydney, Australia; Steven Goodman, Journal of the Society for Clinical Trials, USA; Peter C Gøtzsche, Nordic Cochrane Centre, Denmark; Trish Groves, BMJ; Steven Grunberg, American Society of Clinical Oncology, US; Brian Haynes, McMaster University, Canada; Sally Hopewell, Centre for Statistics in Medicine, University of Oxford; Astrid James, Lancet; Peter Juhn, Johnson \& Johnson, US; Philippa Middleton, University of Adelaide, Australia; Don Minckler, University of California Irvine, US; D Moher, Ottawa Methods Centre, Clinical Epidemiology Program, Ottawa Hospital Research Institute, Canada; Victor M Montori, Knowledge and Encounter Research Unit, Mayo Clinic College of Medicine, US; Cynthia Mulrow, Annals of Internal Medicine, US; Stuart Pocock, London School of Hygiene \& Tropical Medicine; Drummond Rennie, JAMA, US; David L Schriger, Annals of Emergency Medicine, US; KF Schulz, Family Health International, US; Iveta Simera, EQUATOR Network; Elizabeth Wager, Sideview.

Contributors to CONSORT 2010 who did not attend the Montebello meeting: Mike Clarke, UK Cochrane Centre; Gordon Guyatt, McMaster University, Canada.

\section{REFERENCES}

1. Jüni P, Altman DG, Egger M. Systematic reviews in health care: Assessing the quality of controlled clinical trials. $B M J$ 2001;323(7303):42-46.

2. Chan AW, Altman DG. Epidemiology and reporting of randomised trials published in PubMed journals. Lancet 2005;365(9465):1159-1162.

3. Glasziou P, Meats E, Heneghan C, Shepperd S. What is missing from descriptions of treatment in trials and reviews? $B M J$ 2008;336(7659):1472-1474.

4. Dwan K, Altman DG, Arnaiz JA, Bloom J, Chan AW, Cronin E, et al. Systematic review of the empirical evidence of study publication bias and outcome reporting bias. PLoS ONE 2008;3(8):e3081.

5. Begg C, Cho M, Eastwood S, Horton R, Moher D, Olkin I, et al. Improving the quality of reporting of randomized controlled trials. The CONSORT statement. JAMA 1996;276(8):637-639.

6. Moher D, Schulz KF, Altman DG. The CONSORT statement: revised recommendations for improving the quality of reports of parallel-group randomised trials. Lancet 2001;357(9263):1191-1194.

7. Moher D, Schulz KF, Altman DG; CONSORT Group (Consolidated Standards of Reporting Trials). The CONSORT statement: revised recommendations for improving the quality of reports of parallelgroup randomized trials. Ann Intern Med 2001;134(8):657-662.

8. Moher D, Schulz KF, Altman D; CONSORT Group (Consolidated Standards of Reporting Trials). The CONSORT statement: revised recommendations for improving the quality of reports of parallelgroup randomized trials. JAMA 2001;285(15):1987-1991.

9. Plint AC, Moher D, Morrison A, Schulz K, Altman DG, Hill C, et al. Does the CONSORT checklist improve the quality of reports of randomised controlled trials? A systematic review. Med J Aust 2006;185(5):263-267.

10. Hopewell S, Dutton S, Yu L-M, Chan A-W, Altman DG. The quality of reports of randomised trials in 2000 and 2006: a comparative study of articles indexed by PubMed. BMJ 2010;340:c723.

11. Campbell MK, Elbourne DR, Altman DG; CONSORT group. CONSORT statement: extension to cluster randomised trials. BMJ 2004;328(7441):702-708.

12. Piaggio G, Elbourne DR, Altman DG, Pocock SJ, Evans SJW; Consort group. Reporting of noninferiority and equivalence randomized trials: an extension of the CONSORT statement. JAMA 2006;295(10):1152-1160.

13. Moher D, Hopewell S, Schulz KF, Montori V, Gøtzsche PC, Devereaux PJ. CONSORT 2010 Explanation and Elaboration: updated guidelines for reporting parallel group randomised trials. $B M J$ 2010;340:c869.

14. Schulz KF, Chalmers I, Hayes RJ, Altman DG. Empirical evidence of bias. Dimensions of methodological quality associated with estimates of treatment effects in controlled trials. JAMA 1995;273(5):408-412.

15. Moher D, Pham B, Jones A, Cook DJ, Jadad AR, Moher M, et al. Does quality of reports of randomised trials affect estimates of intervention efficacy reported in meta-analyses? Lancet 1998;352(9128):609-613.

16. Moher D, Liberati A, Tetzlaff J, Altman DG; PRISMA Group. Preferred reporting items for systematic reviews and meta-analyses: the PRISMA statement. BMJ 2009;339:b2535.

17. Bossuyt PM, Reitsma JB, Bruns DE, Gatsonis CA, Glasziou PP, Irwig LM, et al. Towards complete and accurate reporting of studies of diagnostic accuracy: the STARD initiative. BMJ 2003;326(7379):41-44.

18. von Elm E, Altman DG, Egger M, Pocock SJ, Gøtzsche PC, Vandenbroucke JP; STROBE Initiative. Strengthening the reporting of observational studies in epidemiology (STROBE) statement: guidelines for reporting observational studies. BMJ 2007;335(7624):806-8.

19. Standards of Reporting Trials Group. A proposal for structured reporting of randomized controlled trials. The Standards of Reporting Trials Group. JAMA 1994;272(24):1926-1931.

20. Rennie D. Reporting randomized controlled trials. An experiment and a call for responses from readers. JAMA 1995;273(13):10541055 .

21. Hopewell S, Clarke M, Moher D, Wager E, Middleton P, Altman DG, et al. CONSORT for reporting randomised trials in journal and conference abstracts. Lancet 2008;371(9609):281-283.

22. Chan AW, Hróbjartsson A, Haahr MT, Gøtzsche PC, Altman DG. Empirical evidence for selective reporting of outcomes in randomized trials: comparison of protocols to published articles. JAMA 2004;291(20):2457-2465.

23. Sackett DL. Commentary: Measuring the success of blinding in RCTs: don't, must, can't or needn't? Int $J$ Epidemiol 2007;36(3):664-665.

24. Schulz KF, Grimes DA. Blinding in randomised trials: hiding who got what. Lancet 2002;359(9307):696-700.

25. Montori VM, Devereaux PJ, Adhikari NK, Burns KE, Eggert CH, Briel M, et al. Randomized trials stopped early for benefit: a systematic review. JAMA 2005;294(17):2203-2209.

26. Hollis S, Campbell F. What is meant by intention to treat analysis? Survey of published randomised controlled trials. BMJ 1999;319(7211):670-674. 
27. Nuovo J, Melnikow J, Chang D. Reporting number needed to treat and absolute risk reduction in randomized controlled trials. JAMA 2002;287(21):2813-2814.

28. Ioannidis JPA, Evans SJW, Gøtzsche PC, O’Neill RT, Altman DG, Schulz K, et al. Better reporting of harms in randomized trials: an extension of the CONSORT statement. Ann Intern Med 2004;141(10):781-788.

29. De Angelis C, Drazen JM, Frizelle FA, Haug C, Hoey J, Horton $\mathrm{R}$, et al. Clinical trial registration: a statement from the International Committee of Medical Journal Editors. Lancet 2004;364(9438):911-912.

30. Lexchin J, Bero LA, Djulbegovic B, Clark O. Pharmaceutical industry sponsorship and research outcome and quality: systematic review. $B M J$ 2003;326(7400):1167-1170.

31. Hopewell S, Clarke M, Moher D, Wager E, Middleton P, Altman DG, et al. CONSORT for reporting randomized controlled trials in journal and conference abstracts: explanation and elaboration. PLoS Med 2008;5(1):e20.

32. Boutron I, Moher D, Altman DG, Schulz KF, Ravaud P; CONSORT group. Extending the CONSORT statement to randomized trials of nonpharmacologic treatment: explanation and elaboration. Ann Intern Med 2008;148(4):295-309.

33. Gagnier JJ, Boon H, Rochon P, Moher D, Barnes J, Bombardier C; CONSORT group. Reporting randomized, controlled trials of herbal interventions: an elaborated CONSORT statement. Ann Intern Med 2006;144(5):364-367.

34. Zwarenstein M, Treweek S, Gagnier JJ, Altman DG, Tunis S, Haynes B, et al. Improving the reporting of pragmatic trials: an extension of the CONSORT statement. BMJ 2008;337:a2390.
Citation: Schulz KF, Altman DG, Moher D; for the CONSORT Group. CONSORT 2010 Statement: updated guidelines for reporting parallel group randomized trials. Open Med 2010;4(1):60-68.

\section{Published: 24 March 2010}

Copyright: Open Medicine applies the Creative Commons Attribution Share Alike License, which means that authors retain copyright of their work and that anyone is able to freely copy, distribute, transmit or adapt the work as long as all adapted or derivative work is distributed under the same or similar licence to this one and all derivative or non-derivative work is attributed to the author and to Open Medicine as specified by the author or Open Medicine but in no way that suggests that the author or Open Medicine endorses the work or how it is used. Any of these conditions can be waived with permission from the copyright holder. These conditions do not negate or supersede fair use laws in any country. For more information, please see our Creative Commons Attribution ShareAlike 2.5 Canada Licence

Note: To encourage dissemination of the CONSORT 2010 Statement, this article is freely accessible on the Open Medicine website and on bmj.com and will also be published in the Lancet, Obstetrics and Gynecology, PLoS Medicine, Annals of Internal Medicine, Journal of Clinical Epidemiology, BMC Medicine and Trials. The authors jointly hold the copyright of this article. For details on further use, see the CONSORT website (www.consort-statement.org). 